\title{
A Multiband Printed Log-Periodic Dipole Array for Wireless Communications
}

\author{
Giovanni Andrea Casula and Paolo Maxia \\ Dipartimento di Ingegneria Elettrica ed Elettronica, Università di Cagliari, Piazza D’Armi, 09123 Cagliari, Italy
}

Correspondence should be addressed to Giovanni Andrea Casula; a.casula@diee.unica.it

Received 8 May 2014; Accepted 4 July 2014; Published 15 July 2014

Academic Editor: Renato Cicchetti

Copyright ( 2014 G. A. Casula and P. Maxia. This is an open access article distributed under the Creative Commons Attribution License, which permits unrestricted use, distribution, and reproduction in any medium, provided the original work is properly cited.

\begin{abstract}
A multiband printed Log-periodic dipole array (LPDA) antenna for wireless communications is presented. The antenna has been designed starting from Carrel's theory, optimized using CST Microwave Studio 2012, and then realized. The comparison between simulated and measured results shows that the proposed antenna can be used for wireless communications both in the S (2.4$3 \mathrm{GHz})$ and in the $\mathrm{C}(5.2-5.8 \mathrm{GHz})$ frequency bands, with very good input matching and a satisfactory end-fire radiation pattern. Moreover, it has a compact size, is very easy to realize, and presents an excellent out-of-band rejection, without the use of stop-band filters, thus avoiding interference out of its operating frequency band.
\end{abstract}

\section{Introduction}

The increasing demands of wireless, and short range, high data rate transmissions, pushed to propose new wireless protocols using different bands of the frequency spectrum, in order to support high data rate wireless communications. This rapid development of short-range radio links in the mobile communications and wireless industry (especially Wi-Fi and wireless local area network (WLAN)) calls for antennas able to operate in different frequency bands simultaneously (multiband antennas), offering wideband operations covering the whole WLAN services. The most common desirable requirement consists of providing multiband operations, and the frequency bands required to a single antenna are $2.4-2.484 \mathrm{GHz}$ for Bluetooth applications, $2.4 \mathrm{GHz}$ and $5 \mathrm{GHz}$ for Wi-Fi applications (following HiperLan protocol), and $2.4 \mathrm{GHz}, 5.2 \mathrm{GHz}, 5.4$ and $5.8 \mathrm{GHz}$ for WLAN applications (following WLAN IEEE 802.11 standards). The demand for antennas offering high performance, compact size, and low cost, besides an easy integration into frontend circuits, suggests the use of printed technologies [13]. As a matter of fact, planar antennas are widely used because of their low profiles, easy design, and fabrication. In the design of the planar antennas, the microstrip-fed [46] and coplanar waveguide (CPW-) fed [7-10] are the most popular feeding structures adopted in the recent literature. Multifrequency antennas are becoming very important since their use allows the reduction of the numbers of antennas and the meeting of the applications of many different wireless communication systems simultaneously, such as Wireless Local Area Network (WLAN) and IEEE 802.16 Worldwide Interoperability for Microwave Access (WiMAX). A variety of structures for designing multiband WLAN planar antennas have been proposed in recent years [4-12], based on known antenna concepts, but showing either a multiband or a tunable behaviour. Among the available current wireless communication standards, $\mathrm{Wi}-\mathrm{Fi}$ is nowadays rapidly gaining more and more supporters. The Wi-Fi standard is based on the well-established protocols IEEE 802.11a, 802.11b, and 802.11 g and the emerging 802.11n [13]. The considered operating frequencies are within the industrial, scientific, and medical (ISM) free window: 2.412 and $2.484 \mathrm{GHz}(2.45-\mathrm{GHz}$ center frequency and $72-\mathrm{MHz}$ bandwidth) for the $802.11 \mathrm{~b}$ and $802.11 \mathrm{~g}$ protocols, 5.170 , and $5.805 \mathrm{GHz}$ (5.5- $\mathrm{GHz}$ center frequency and 635-MHz bandwidth) for the 802.11a protocol, while the 802.11n protocol employs both frequency bands simultaneously $[13,14]$.

In this work, a multiband printed log-periodic dipole array, working both in the $\mathrm{S}$ and in the $\mathrm{C}$ frequency bands (from 2.4 to $3 \mathrm{GHz}$ and from 5.2 to $5.8 \mathrm{GHz}$ ), which can be 


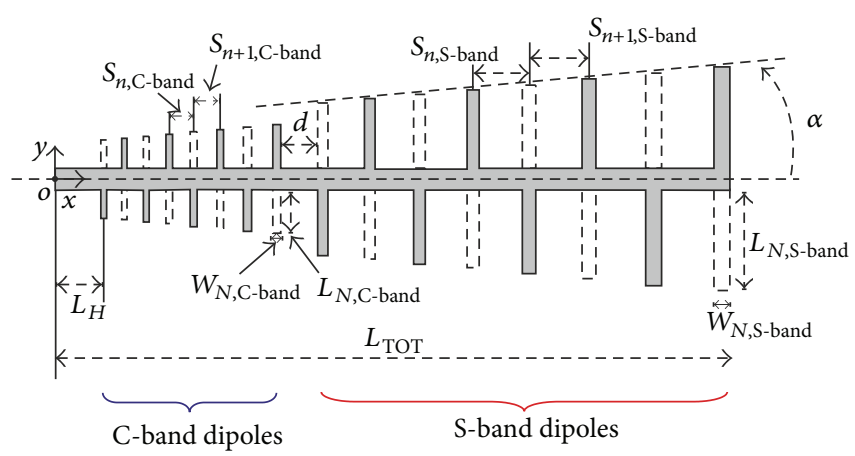

(a)

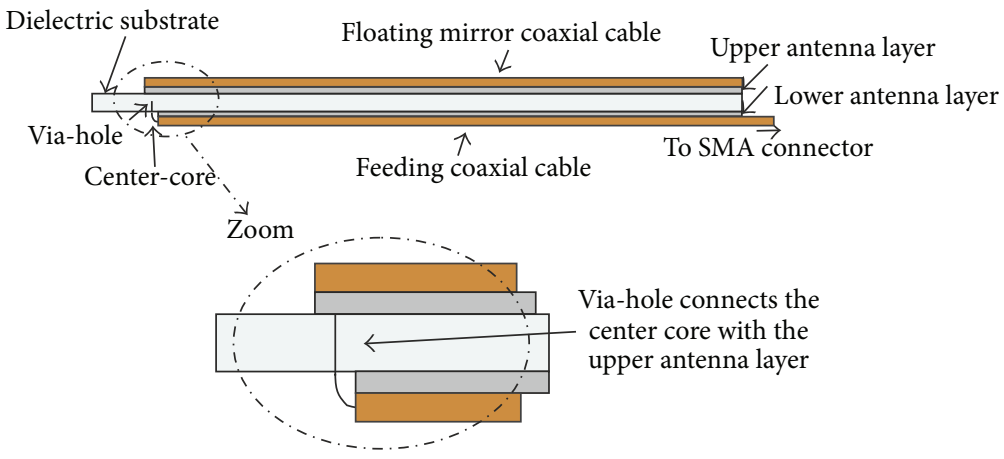

(b)

FIGURE 1: S-C band feed layout. $L_{\mathrm{TOT}}=124 \mathrm{~mm}, L_{H}=8.962 \mathrm{~mm}$. (a) Top view; (b) side view (with feeding and mirror coaxial cables).

used as a multiband antenna for wireless communications, is presented, satisfying the requirements of several wireless communication standards, such as HiperLan, IEEE 802.11 and Bluetooth [13]. The proposed antenna is very easy to realize, is very compact, and presents an excellent out-ofband rejection, without the use of stop-band filters. The designed antenna meets also the requirements of meteorological radars, whose operating frequency bands are $2.7-3.0 \mathrm{GHz}$ for the S-band and 5.4-5.8 GHz for the C-band [1]. Therefore, it can be effectively used also as a feed for reflector antennas in weather radar applications.

\section{Antenna Design}

In this section the design of a high gain feed for wireless communications is presented. As pointed out in Section 1, the S- and C-band are the most widely used in wireless communications, and therefore the design of a high gain printed log periodic feed able to work both in S- and C-band is discussed here. The concept of log-periodic printed antennas is applied separately to two different groups of dipoles designed to operate each one in a specific frequency band. The two groups of dipoles have been then connected together, obtaining the configuration shown in Figure 1. The distance $d$ between the two groups of dipoles has subsequently been optimized, aiming for the best input matching of the whole antenna. The final distance $d$ is relatively small, and comparable to the spacing between two adjacent dipoles of the LPDA, resulting in a very compact multiband antenna.
This solution allows the obtaining of a log periodic antenna with a reduced size, operating only in the ranges 2.4-3.0 GHz and 5.2-5.8 GHz, instead of a complete printed LPDA array working between 2.4 and $5.8 \mathrm{GHz}$.

2.1. S-Band and C-Band LPDA Design. The two LPDAs operating, respectively, only in the S-band and only in the C-band have been designed separately, and their geometrical parameters are shown in Table 1. For each group of dipoles, the number of dipoles and the scaling factor $\tau$ must be defined [15-17]. Even though both parameters can be different for each group of dipoles, as a design rule we choose to use the same values for both groups.

The number of dipoles of each group is determined by the design specifications (i.e., the requirements on the frequency bandwidth and the directivity). In our case, for the proposed printed LPDA feed, we require an average directivity of $9 \mathrm{dBi}$, and therefore, following Carrel [15], we set the log period, $\tau=$ $L_{n+1} / L_{n}$, and the spacing factor, $\sigma=S_{n} / 4 L_{n}$, of both groups of dipoles to the values $\tau=0.94$ and $\sigma=0.17$.

The chosen dielectric substrate is the ARLON AD 450, a material developed for high power applications [18], with low losses (dielectric loss tangent $\delta=0.0035$ ) and a dielectric permittivity $\varepsilon_{r}=4.5$. The substrate thickness and metallization are, respectively, $h=1.524 \mathrm{~mm}$ and $t=$ $0.070 \mathrm{~mm}$. The metal thickness has been chosen to be twice the typical metal thickness of LPDAs $(0.035 \mathrm{~mm})$, so as to increase the power level capability of the antenna. 
TABLE 1: Geometrical parameters of S-C band printed LPDA.

\begin{tabular}{lccc}
\hline Dipole & $L_{n}[\mathrm{~mm}]$ & $W_{n}[\mathrm{~mm}]$ & $S_{n}[\mathrm{~mm}]$ \\
\hline $8_{\text {S-band }}$ & 18.44 & 2.87 & 12.54 \\
$7_{\text {S-band }}$ & 17.34 & 2.70 & 11.79 \\
$6_{\text {S-band }}$ & 16.30 & 2.54 & 11.08 \\
$5_{\text {S-band }}$ & 15.32 & 2.39 & 10.42 \\
$4_{\text {S-band }}$ & 14.40 & 2.24 & 9.79 \\
$3_{\text {S-band }}$ & 13.54 & 2.11 & 9.20 \\
$2_{\text {S-band }}$ & 12.72 & 1.98 & 8.65 \\
$1_{\text {S-band }}$ & 11.96 & 1.86 & \\
\hline $8_{\text {C-band }}$ & 7.99 & 1.50 & 5.43 \\
$7_{\text {C-band }}$ & 7.51 & 1.41 & 5.10 \\
$6_{\text {C-band }}$ & 7.06 & 1.32 & 4.80 \\
$5_{\text {C-band }}$ & 6.64 & 1.25 & 4.51 \\
$4_{\text {C-band }}$ & 6.24 & 1.17 & 4.24 \\
$3_{\text {C-band }}$ & 5.86 & 1.10 & 3.99 \\
$2_{\text {C-band }}$ & 5.51 & 1.03 & 3.74 \\
$1_{\text {C-band }}$ & 5.18 & 0.97 & \\
\hline
\end{tabular}

The characteristic impedance $Z_{0}$ of the printed feeding lines (paired strips) of the two groups of dipoles has been selected in order to obtain easy matching with the employed UT- 056 coaxial cables [19]: by choosing $Z_{0}=50 \Omega$, we obtain $W=2.8864 \mathrm{~mm}$.

The number $N$ of elements of each group of dipoles is computed by using the expressions given by Carrel [15-17]; starting from the required bandwidths, we get number of dipoles $N=8$ and aperture angle $2 \alpha=5.042^{\circ}$.

The lengths $L_{N, S-B a n d}$ and $L_{N, C-B a n d}$ and the widths $W_{N, S-B a n d}$ and $W_{N, C-B a n d}$ of the longest dipole of each group (S-band and C-band) have been evaluated using the cut-andtry procedure described in $[16,17]$, obtaining $L_{N, S-B a n d}=$ $18.444 \mathrm{~mm}, W_{N, S-B a n d}=2.875 \mathrm{~mm}, L_{N, \mathrm{C}-\text { Band }}=7.990 \mathrm{~mm}$, and $W_{N, C-B a n d}=1.50 \mathrm{~mm}$.

The lengths and widths of the other dipoles of each group are computed by using the well-known expressions for LPDAs $[16,17,20]$ :

$$
\frac{L_{n+1}}{L_{n}}=\frac{1}{\tau} \quad n=1, \ldots, 8 .
$$

In Figure 2 the simulated reflection coefficient for the group of dipoles designed to work in the S-band is reported, and the input matching is less than $-10 \mathrm{~dB}$ in the required frequency band $2.4-3 \mathrm{GHz}$. Figure 3 shows the simulated reflection coefficient for the group of dipoles designed to work in the $\mathrm{C}$-band, and, also in this case, the input matching is less than $-10 \mathrm{~dB}$ in the required frequency band $(5.2-5.8 \mathrm{GHz})$.

Figures 2 and 3 show also the simulated realized gain $G_{R}$ for the group of dipoles working in the S- and C-band, respectively. In both cases, the realized gain rapidly decreases out of the working bandwidth, while the radiation pattern deteriorates in the same way, showing a higher SLL and a bad front-to-back ratio with respect to the values assumed within the antenna working bandwidth.

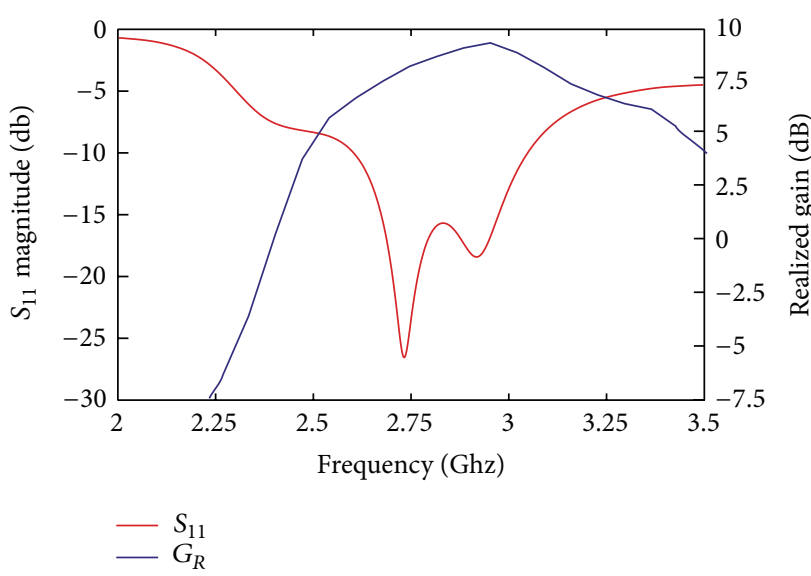

FIGURE 2: S-band dipoles group reflection coefficient and realized gain versus frequency.

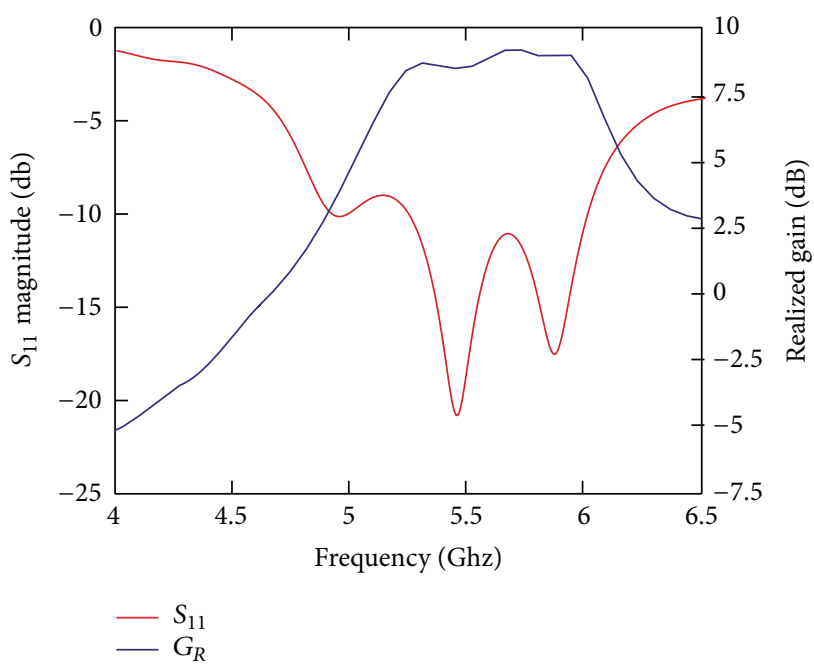

FIGURE 3: C-band dipoles group reflection coefficient and realized gain.

2.2. Complete Antenna Design. The two groups of dipoles designed in Section 2.1 have been connected together, obtaining the configuration shown in Figure 1. The geometrical parameters of the dipoles are reported in Table 1.

The value of the distance $d$ between the two groups of dipoles has been chosen so to obtain the best input matching of the whole antenna. The distance $d$ has been optimized using CST Microwave Studio, and the optimal value is equal to only $8.3 \mathrm{~mm}$. This distance is relatively small, and comparable to the spacing between two adjacent dipoles of the LPDA, resulting in a very compact multiband antenna.

The starting length of the final termination $L_{H}$ of the paired strips has been chosen to be equal to one half of the free-space wavelength at the highest operating frequency and then optimized aiming at the best antenna input matching, obtaining the value of $L_{H}=7.374 \mathrm{~mm}$.

The feeding network selected for the designed antenna consists of a coaxial cable. The outer conductor of the coaxial cable is soldered to the bottom layer of the LPDA, and 


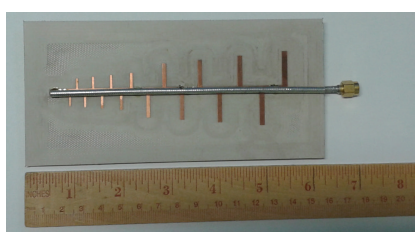

(a)

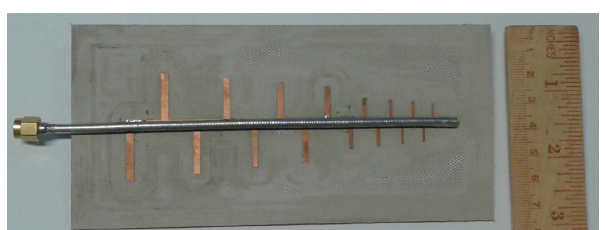

(b)

FIgURE 4: Photo of the designed LPDA antenna shown in Figure 1: (a) front; (b) back.

the inner conductor is connected to the top layer of the antenna using a via-hole inside the substrate. An additional floating mirror coaxial cable, soldered in the top layer of the array, able to improve the antenna performances, has been used (as indicated in Figure 1). In fact, the insertion of an additional mirror coaxial cable gives to the antenna significantly better radiation performances, and allows the stabilizing of the phase center, without affecting the antenna input matching [16]. On the other hand, the LPDA could also be fed using a fully planar network, namely a coplanar waveguide [17], solution which allows a more simple realization, and with a low cost, a compact size, and an easier connection with the SMA connector, but handling only low power levels, due to the dielectric breakdown of the air between the metallic strips.

\section{Results}

The LPDA, designed in Section 2, has been manufactured (see Figure 4) and fully characterized. All the numerical results take into account also the additional floating mirror coaxial cable, positioned in the top layer of the array.

In Figure 5 the comparison between the simulated and experimental reflection coefficient for the complete antenna is shown, and the input matching is very satisfactory, being less than $-10 \mathrm{~dB}$ both in the $\mathrm{S}$ - and in the C-band $(2.4-3 \mathrm{GHz}$, $5.2-5.8 \mathrm{GHz}$ ). The simulated and measured data are in very good agreement, and the out-of-band rejection is very good, especially considering that no stop-band filters have been used in the antenna design.

Figure 6 reports the frequency behaviour of the realized gain $G_{R}$ for the antenna shown in Figure 1 (both evaluated by CST and measured). The antenna gain has an average value equal to $8.75 \mathrm{~dB}$ in the $\mathrm{S}$-band and equal to $9.35 \mathrm{~dB}$ in the Cband. On the other hand, it rapidly drops to less than $3 \mathrm{~dB}$ out of the working frequency band, confirming the very good out-of-band rejection of the proposed antenna.

In Figure 7 the simulated $E$ - and $H$-Plane antenna radiation patterns are shown. The cross-polar component is not shown, since it is always below $-35 \mathrm{~dB}$ with respect to the copolar component of the radiated field. The radiation pattern shows an end-fire behavior within the design frequency band (2.4-3 GHz and 5.2-5.8 GHz), with a SLL below $-27 \mathrm{~dB}$ and an $\mathrm{F} / \mathrm{B}$ ratio above $28 \mathrm{~dB}$, while it deteriorates very rapidly out-of-band, with both a bad SLL and front-to-back ratio. The symmetry of the in-band radiated field is very good both in the $E$ - and in the $H$-planes, thanks to the additional

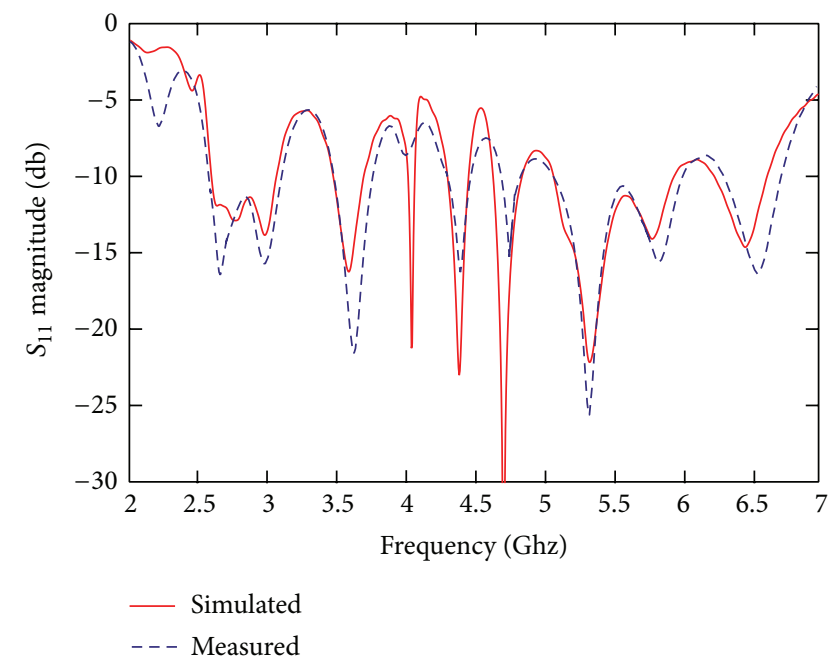

FIGURE 5: Magnitude of the reflection coefficient versus frequency for the designed LPDA antenna shown in Figure 1.

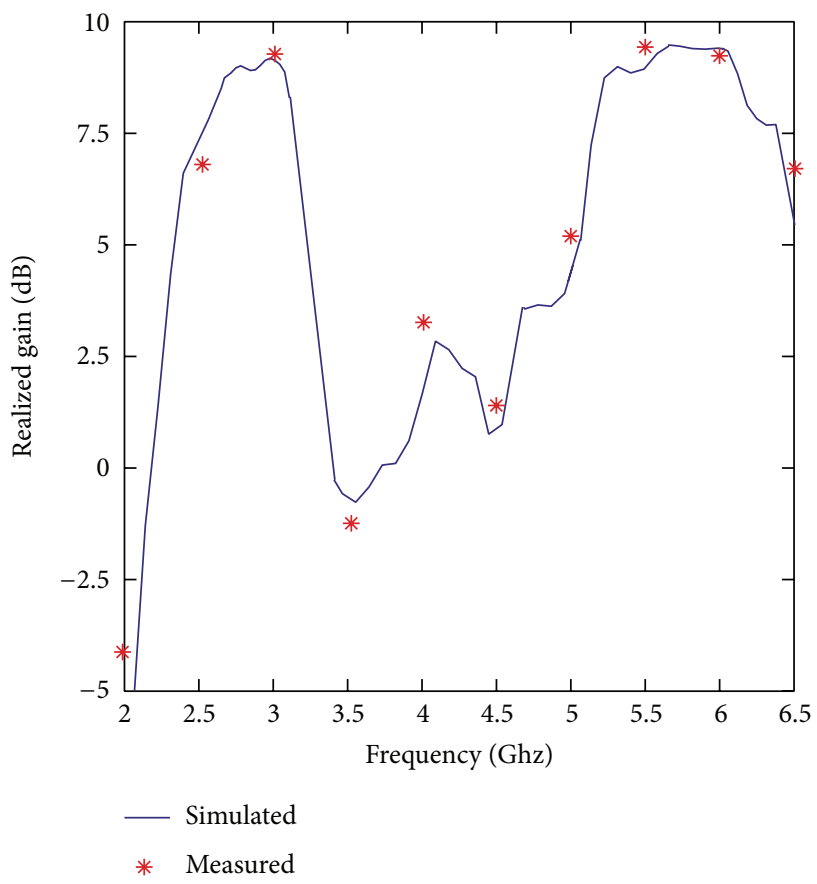

FIGURE 6: Realized gain for the designed LPDA antenna shown in Figure 1 . 

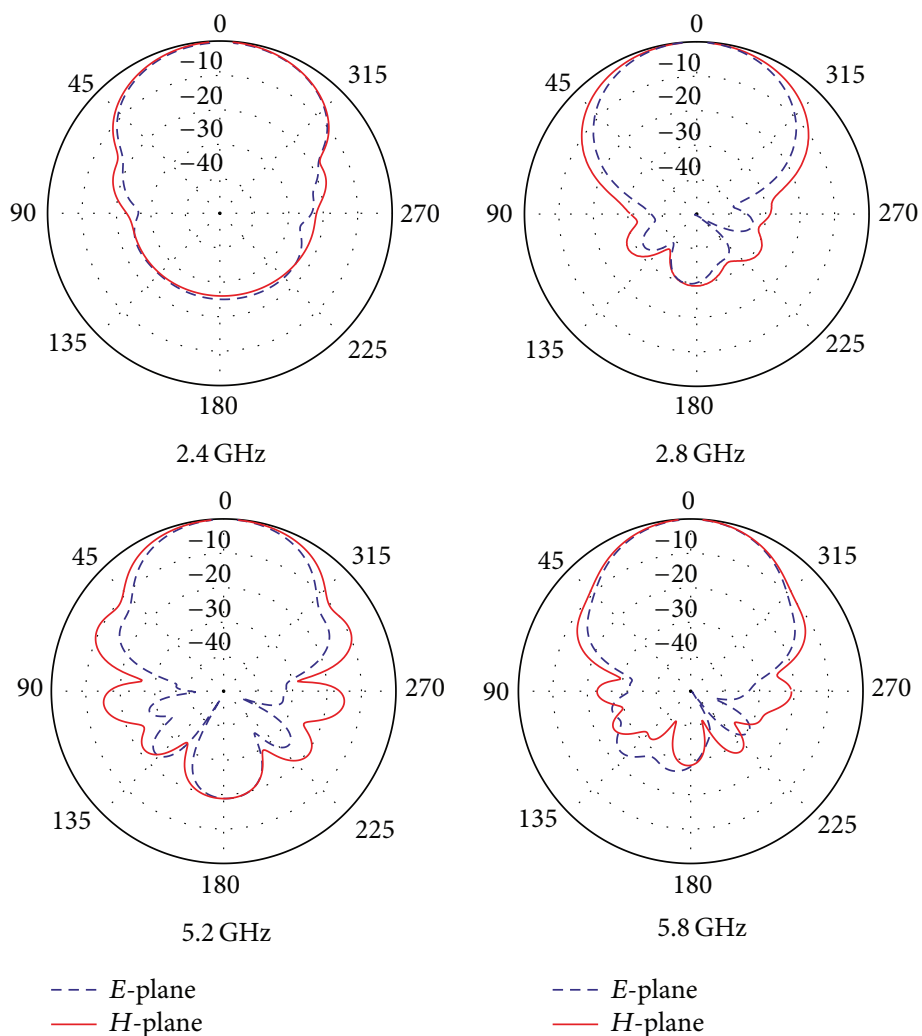

(a)
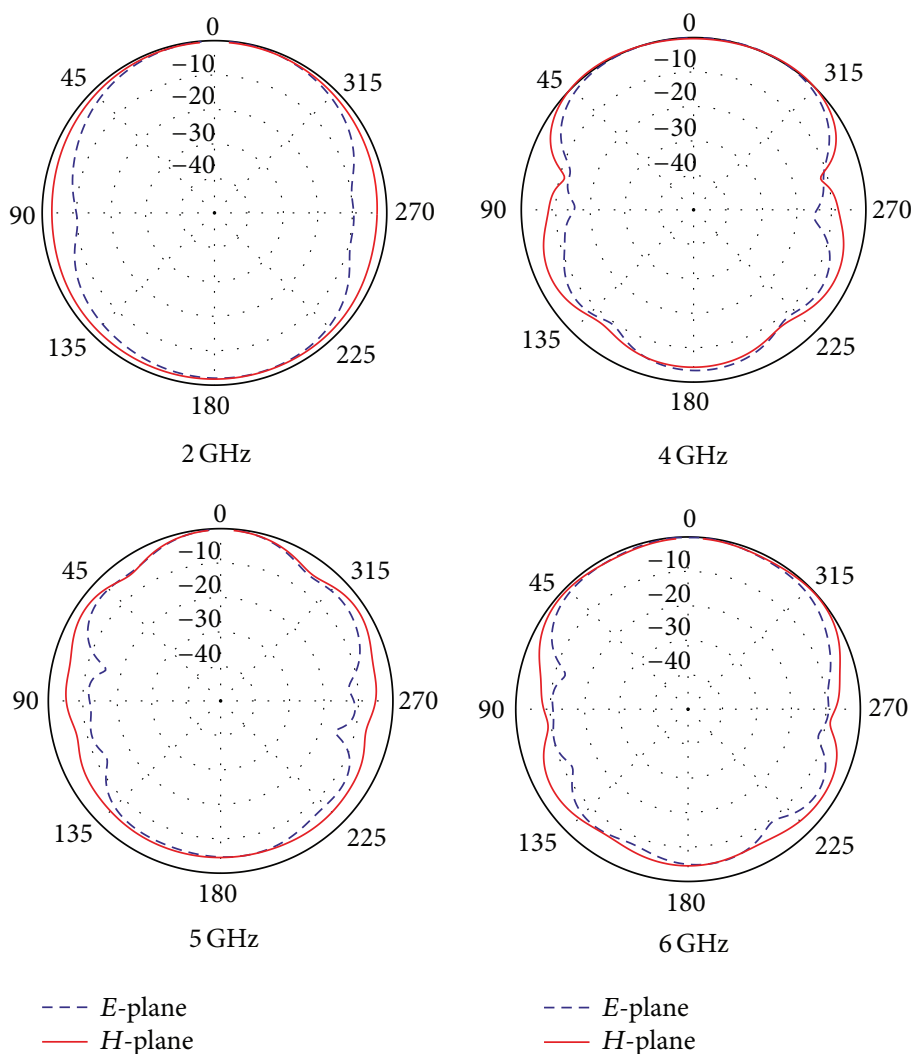

(b)

Figure 7: E-Plane and $H$-Plane radiation pattern of the designed LPDA antenna shown in Figure 1: (a) in-band; (b) out-of-band. 
mirror coaxial cable, soldered in the top layer of the LPDA. Therefore, the proposed LPDA can be successfully used as a multiband antenna for wireless communications.

\section{Conclusion}

A multiband printed Log-periodic dipole array (LPDA) antenna for wireless communications, covering both the $S$ $(2.4-3 \mathrm{GHz})$ and the $\mathrm{C}(5.2-5.8 \mathrm{GHz})$ frequency bands has been presented. The antenna is fed using two coaxial cables, which provide the required broadband input matching, and improve the radiation pattern when compared with an antenna fed with a single coaxial cable. The simulated and measured results are in very good agreement, showing a very good input matching, an end-fire radiation pattern, and an excellent rejection out of its operating frequency band, without the use of stop-band filters, avoiding undesired interference. The antenna realized gain is above $8.75 \mathrm{~dB}$ within the working band, decreasing to less than $3 \mathrm{~dB}$ in the out-of-band range.

\section{Conflict of Interests}

The authors declare that there is no conflict of interests.

\section{References}

[1] P. Callaghan and J. C. Batchelor, "Dual-band pin-patch antenna for Wi-Fi applications," IEEE Antennas and Wireless Propagation Letters, vol. 7, pp. 757-760, 2008.

[2] Y. J. Cho, Y. S. Shin, S. H. Hwang, and S.-O. Park, "A wideband internal antenna with dual monopole radiation elements," IEEE Antennas and Wireless Propagation Letters, vol. 4, no. 1, pp. 381384, 2005.

[3] R. K. Joshi and A. R. Harish, "A modified bow-tie antenna for dual band applications," IEEE Antennas and Wireless Propagation Letters, vol. 6, pp. 468-471, 2007.

[4] X. Q. Zhang, Y. C. Jiao, and W. H. Wang, "Compact wide triband slot antenna for WLAN/WiMAX applications," Electronics Letters, vol. 48, no. 2, pp. 64-65, 2012.

[5] C.-C. Lin, L.-C. Kuo, and H.-R. Chuang, "A horizontally polarized omnidirectional printed antenna for WLAN applications," IEEE Transactions on Antennas and Propagation, vol. 54, no. 11, pp. 3551-3556, 2006.

[6] G. Cappelletti, D. Caratelli, R. Cicchetti, and M. Simeoni, "A low-profile printed drop-shaped dipole antenna for wideband wireless applications," IEEE Transactions on Antennas and Propagation, vol. 59, no. 10, pp. 3526-3535, 2011.

[7] R. K. Raj, M. Joseph, C. K. Aanandan, K. Vasudevan, and P. Mohanan, "A new compact microstrip-fed dual-band coplanar antenna for WLAN applications," IEEE Transactions on Antennas and Propagation, vol. 54, no. 12, pp. 3755-3762, 2006.

[8] H. Chen, X. Yang, Y. Z. Yin, J. J. Wu, and Y. M. Cai, “Tri-band rectangle-loaded monopole antenna with inverted-L slot for WLAN/WiMAX applications," Electronics Letters, vol. 49, no. 20, pp. 1261-1262, 2013.

[9] P. Liu, Y. Zou, B. Xie, X. Liu, and B. Sun, "Compact CPWfed tri-band printed antenna with meandering split-ring slot for WLAN/WiMAX applications," IEEE Antennas and Wireless Propagation Letters, vol. 11, pp. 1242-1244, 2012.
[10] M. J. Hua, P. Wang, Y. Zheng, and S. L. Yuan, "Compact tri-band CPW-fed antenna for WLAN/WiMAX applications," Electronics Letters, vol. 49, no. 18, pp. 1118-1119, 2013.

[11] Y.-L. Kuo and K.-L. Wong, "Printed double-T monopole antenna for $2.415 .2 \mathrm{GHz}$ dual-band WLAN operations," IEEE Transactions on Antennas and Propagation, vol. 51, no. 9, pp. 2187-2192, 2003.

[12] C. T. Lee and K. L. Wong, "Uniplanar printed coupled-fed PIFA with a band-notching slit for WLAN/WiMAX operation in the laptop computer," IEEE Transactions on Antennas and Propagation, vol. 57, no. 4, pp. 1252-1258, 2009.

[13] A. Doufexi, S. Armour, M. Butler et al., "A comparison of the HIPERLAN/2 and IEEE 802.11a wireless LAN standards," IEEE Communications Magazine, vol. 40, no. 5, pp. 172-180, 2002.

[14] D. Caratelli, R. Cicchetti, G. Bit-Babik, and A. Faraone, "A perturbed E-shaped patch antenna for wideband WLAN applications," IEEE Transactions on Antennas and Propagation, vol. 54, no. 6, pp. 1871-1874, 2006.

[15] R. Carrel, "The design of log-periodic dipole antennas," in Proceedings of the IRE International Convention Record, vol. 9, pp. 61-75, New York, NY, USA, March 1961.

[16] G. A. Casula, P. Maxia, G. Mazzarella, and G. Montisci, "Design of a printed log-periodic dipole array for ultra-wideband applications," Progress In Electromagnetics Research C, vol. 38, pp. 15-26, 2013.

[17] G. A. Casula, P. Maxia, G. Montisci, G. Mazzarella, and F. Gaudiomonte, "A printed LPDA fed by a Coplanar waveguide for broadband applications," IEEE Antennas and Wireless Propagation Letters, vol. 12, pp. 1232-1235, 2013.

[18] https://imageserv1l.team-logic.com/mediaLibrary/303/AD450 .pdf.

[19] http://www.micro-coax.com/products/product-details/?type= semi_rigid\&part_id=14.

[20] E. Ávila-Navarro, J. M. Blanes, J. A. Carrasco, C. Reig, and E. A. Navarro, "A new bi-faced log periodic printed antenna," Microwave and Optical Technology Letters, vol. 48, no. 2, pp. 402-405, 2006. 

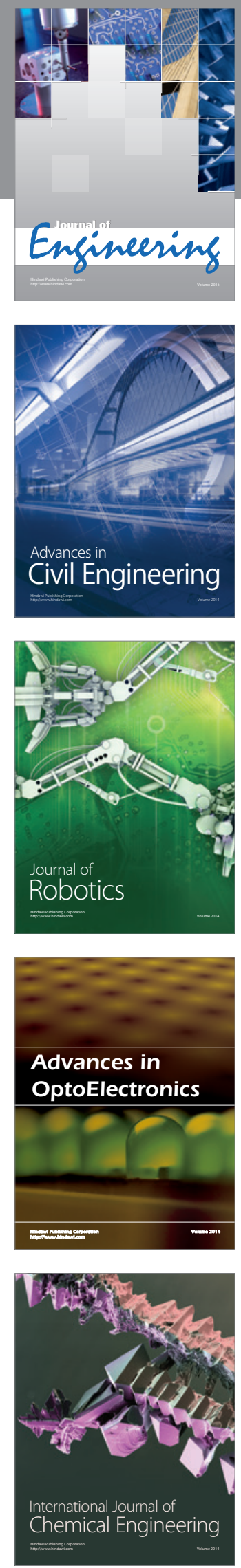

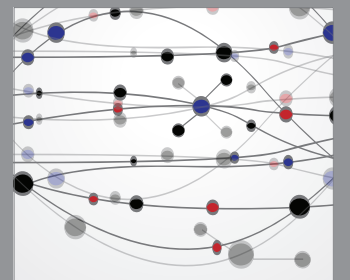

The Scientific World Journal
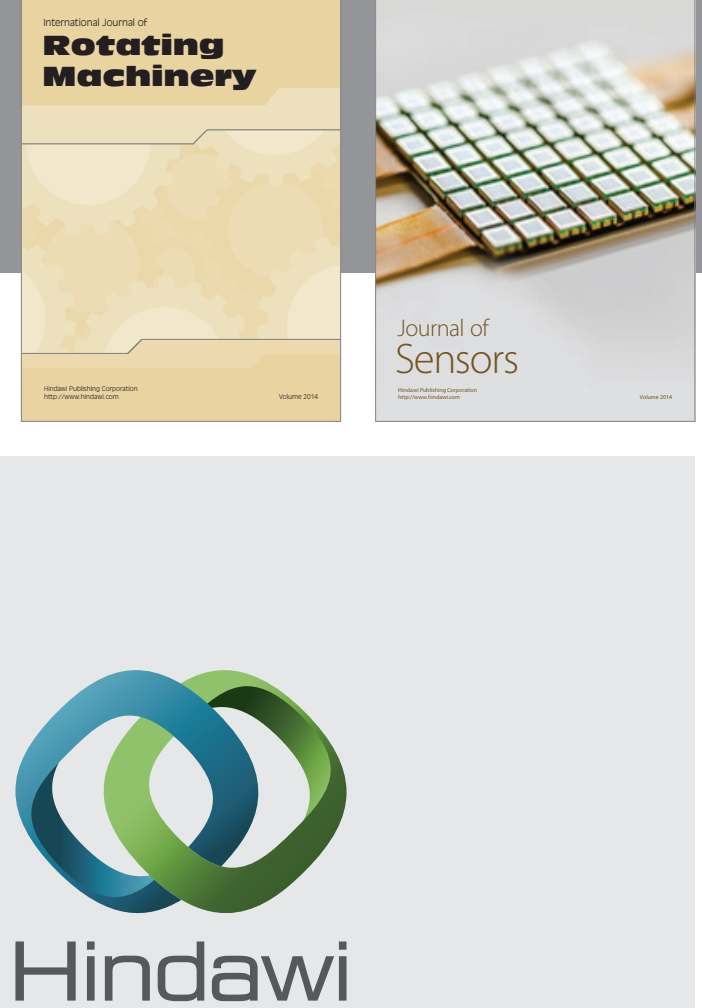

Submit your manuscripts at http://www.hindawi.com
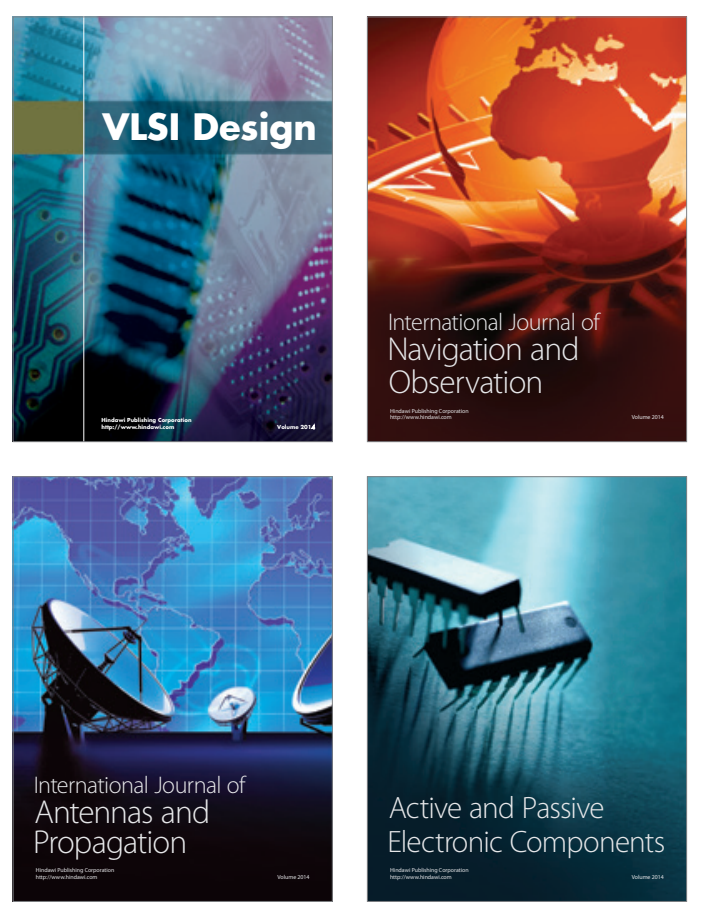
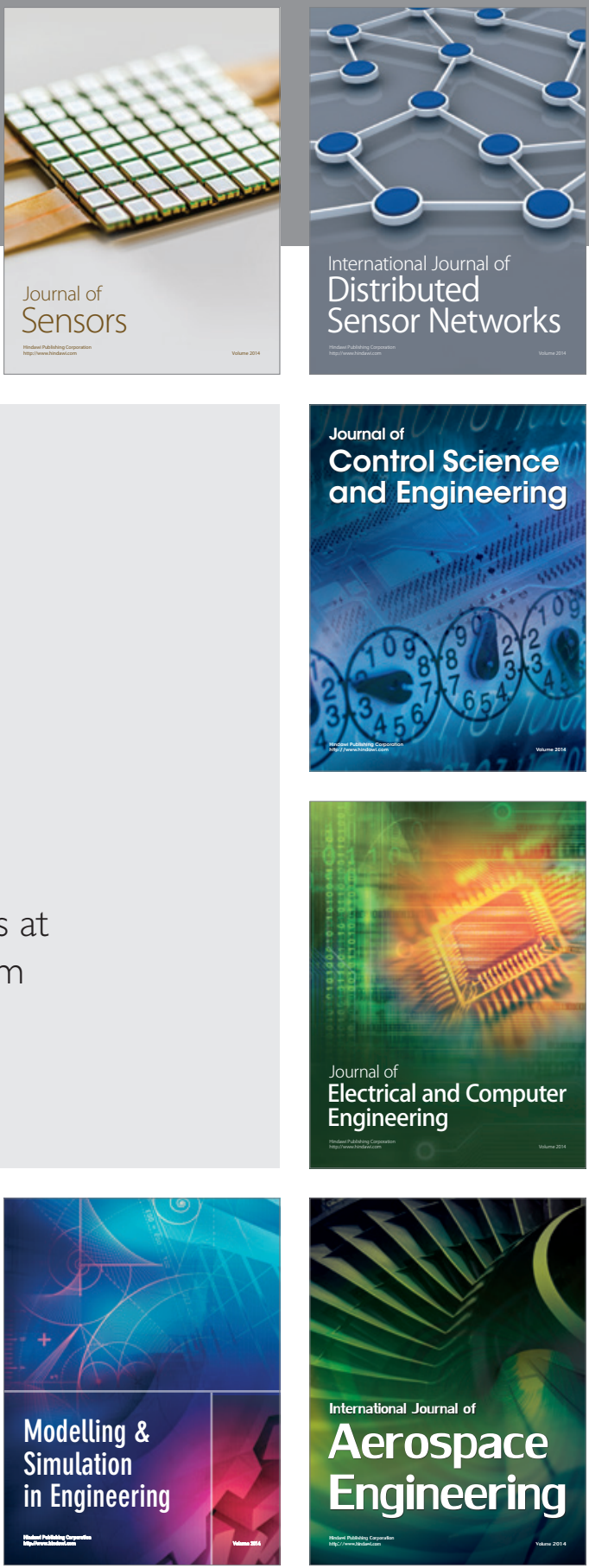

Journal of

Control Science

and Engineering
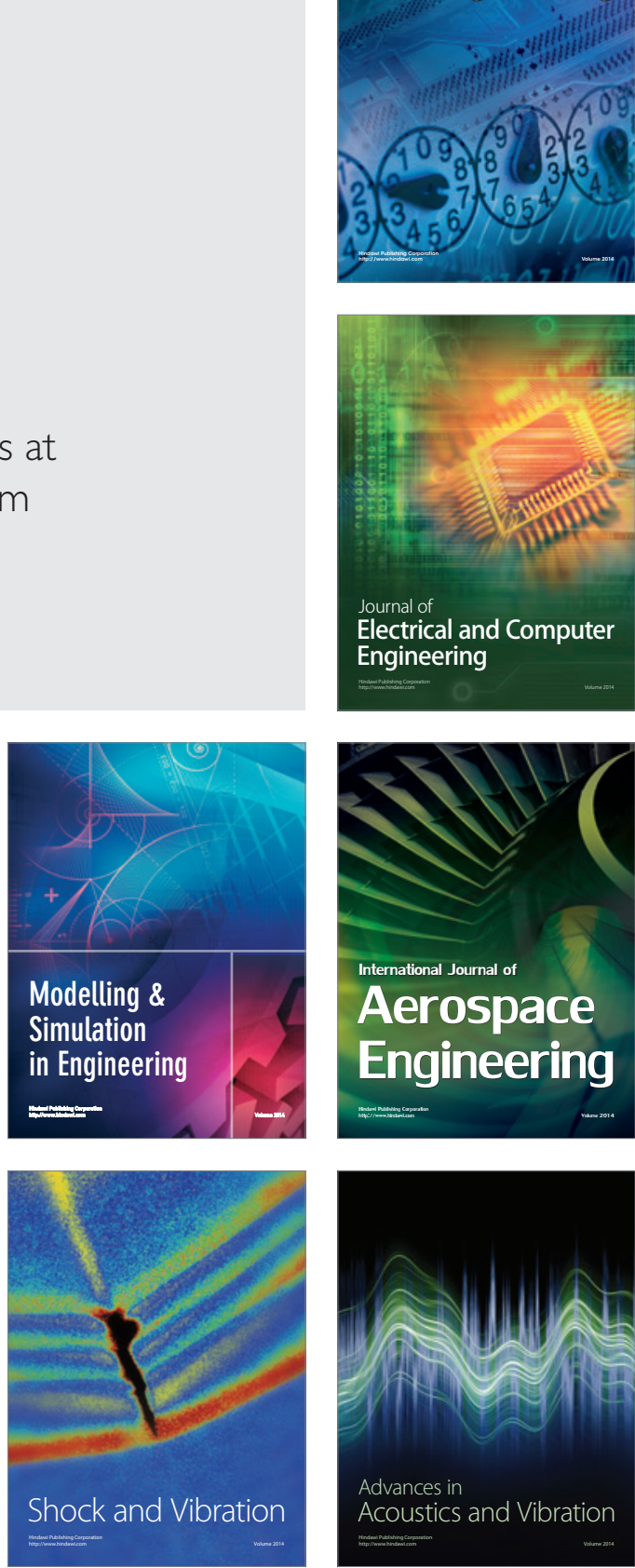